\title{
Commissioning, Performance and Calibration of Crystals of the CMS Electromagnetic Calorimeter
}

\author{
Yong Yang $* \dagger$ \\ California Institute of Technology, Pasadena, CA, USA \\ E-mail: yongy@caltech.edu
}

The operation and general performance of the CMS electromagnetic calorimeter at $\sqrt{s}=7 \mathrm{TeV}$ are described. The first LHC collision data have been used to finalize the commissioning of ECAL readout and trigger and to verify the readiness of ECAL for data taking. Di-photon states of $\pi^{0}$ and $\eta$ have also been used to verify and tune the energy scale. The quality of the offline data reconstruction, from crystal level quanties to clusters, has been investigated and improved using known physics processes. Decays of $\pi^{0}$ into two photons as well as the $\phi$ invariance of the average energy deposition at a given pseudorapidity are utilized to equalize the response of the individual channels. Based on an integrated luminosity of about $250 \mathrm{nb}^{-1}$, an inter-calibration precision of $0.6 \%$ has been achieved in the central barrel ECAL, in the pseudorapidty regions $|\eta|<0.8$.

35th International Conference of High Energy Physics - ICHEP2010,

July 22-28, 2010

Paris France

${ }^{*}$ Speaker.
${ }^{\dagger}$ On behalf of the CMS Collaboration




\section{Overview of the CMS electromagnetic calorimeter}

The electromagnetic calorimeter (ECAL) of the Compact Muon Solenoid (CMS) experiment consists of a barrel section (EB) and two endcaps (EE), using the lead tungstate $\left(\mathrm{PbWO}_{4}\right)$ scintillating crystals as the active medium, with a silicon-strip preshower (ES) in front of each EE. The EB provides the coverage of pseudorapidity $|\eta|<1.479$ with EE extending to $|\eta|=3.0$. The ES covers $1.653<|\eta|<2.6$.

The ECAL energy resolution has been measured in electron test beams to be [1]

$$
\frac{\sigma(E)}{E}=\frac{2.8 \%}{\sqrt{E}(\mathrm{GeV})} \oplus \frac{0.12 \mathrm{GeV}}{E} \oplus 0.3 \%
$$

for electrons incident on the center of crystals. The three contributions correspond to the stochastic, noise and constant terms. In the environment of CMS, for unconverted photons with energies above $100 \mathrm{GeV}$, the energy resolution is dominated by the constant term. As a consequence, the performance of the CMS ECAL at the LHC will depend mainly on the quality of its inter-calibration and monitoring. Achieving the design-goal inter-calibration precision of $0.5 \%$ in situ will be particularly important for a discovery of the Higgs boson in the decay channel $\mathrm{H} \rightarrow \gamma \gamma$, one of the primary goals of the LHC physics program. Besides the inter-calibration, the temperature stability of the crystals/photodetectors and variation of crystal transparency due to radiation also contribute the the constant term. The variation of the crystal transparency is monitored by a laser system, and energy measurement of individual crystal is corrected based on the monitoring data.

\section{Performance of ECAL}

During the first few months of the LHC collisions, the percentage of fully working channels in $\mathrm{EB}$ and $\mathrm{EE}$ is about $99.30 \%$ and $98.94 \%$, respectively. In ES, the percentage of fully-functional strips is $99.79 \%$. The temperature stability over two months is measured to be about $0.0076{ }^{\circ} \mathrm{C}$ and $0.015{ }^{\circ} \mathrm{C}$ for $\mathrm{EB}$ and $\mathrm{EE}$, which meet the specification (maximum variation of $0.05{ }^{\circ} \mathrm{C}$ and $0.1{ }^{\circ} \mathrm{C}$ for EB and EE). The light monitoring system itself shows variation of less than $0.03 \%$, the stability of which is much better than that needed to achieve a constant term in the ECAL energy resolution of $0.5 \%$. In situ timing synchronisation of channels is performed, yielding an average bias of about $50 \mathrm{ps}$, with a spread less than 40 ps. Such precision of timing measurement is important for rejection of backgrounds such as cosmic rays, beam halo muons, electronic noise and out-of-time interactions. Precise time measurement makes it also possible to identify new particles such as photons from decay of long-lived new particles which reach the ECAL out-of-time.

Figure 1 left shows the the energy spectra measured in individual channels of EB from data and Monte Carlo (MC) simulation. The good comparison demonstrates the good performance of ECAL crystals during the first collision at the LHC.

\section{Calibration of ECAL}

The CMS ECAL has been pre-calibrated [2][3] prior to installation with laboratory measurements of crystal light yield and photo-detector gain during the construction phase (all EB and EE 

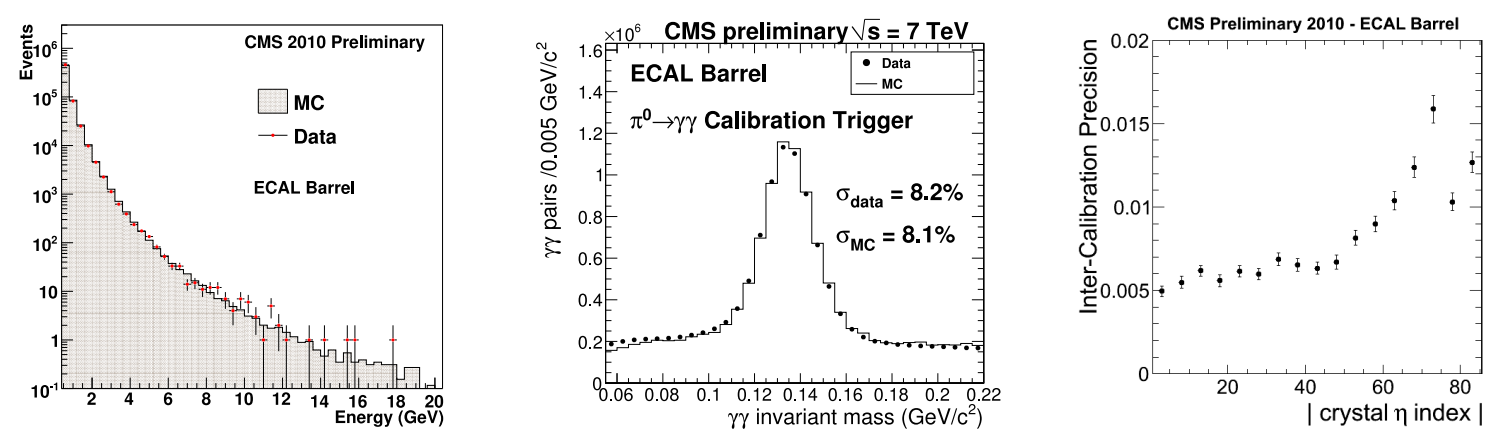

Figure 1: Left, the energy spectra of the individual channels from $7 \mathrm{TeV}$ minimum bias collision events. Center, the reconstructed $\pi^{0}$ invariant mass from a calibration trigger. Right, The obtained precision of combined inter-calibration constants (beam dump, $\pi^{0}$ and $\phi$-symmetry) as a function of crystal pseudorapidity.

channels), with test beam electrons (25\% of EB and about $2.4 \%$ of EE) and with cosmic ray muons (all EB channels). After installation in the LHC, events from the stopped circulated beams (beam dump) have been used to improve the pre-calibration precision, providing an independent intercalibration precision of $1.6 \%$ across the entire barrel. Two methods have been studied with the first data to further improve the precision. First, the $\phi$-symmetry method, based on the assumption that the total transverse energy deposited from minimum bias events should be the same for all crystals in a ring at a fixed pseudorapidity, provides a precision about $1.4 \%$ for crystals in the regions $|\eta|<0.8$. Second, the $\pi^{0}$ calibration method, uniformizing the $\pi^{0}$ peak positions for individual crystals, provides a precision about $1.2 \%$ for the same pseudorapidty regions. The result is consistent with the expectation from MC studies which give $1.1 \pm 0.2 \%$. The systematic limit of the precision of this method has not yet been reached and the current precision is dominated by the available statistics of $250 \mathrm{nb}^{-1}$. Events used for both methods are selected from special designed calibration triggers which can run at much higher rate than the physics rate due to the smaller event size. Figure 1 (center) shows the reconstructed $\pi^{0}$ peak from one such calibration trigger, comparison between data and MC demonstrates the good performance of the ECAL triggers and crystals. Combining with the calibration from the two methods and the beam dump provides a precision about $0.6 \%$ for $|\eta|<0.8$ (Figure 1 right). The absolute energy scale of EB has been verified by the reconstructed $\pi^{0}$ and $\eta$ meson mass peaks in data, and is found to agree with MC at a level of $1 \%$. The inter-calibration of the ECAL crystals will continue to improve with more LHC data, with collection of larger samples of $\pi^{0}$ and $\eta$ mesons decaying into photons as well as $\mathrm{W}$ and $\mathrm{Z}$ bosons decaying into electrons.

\section{References}

[1] CMS Collaboration, The CMS experiment at the CERN LHC, 2008, JINST 0803, S08004.

[2] P. Adzic et al., Intercalibration of the barrel electromagnetic calorimeter of the CMS experiment at start-up, 2008, JINST 3, P10007.

[3] CMS Collaboration, Performance and operation of the CMS electromagnetic calorimeter, 2010, JINST 5, T03010. 